\title{
THE PROBLEM OF PARTIAL GEODESIC REPRESENTATION*
}

BY

\section{EDWARD KASNER}

\section{§1. The general problem.}

The original problem of geodesic representation, solved by DinI (1869) with reality restrictions and by LIE (1883) in complete generality, may be stated as follows : Find all pairs of surfaces $S$ and $S_{1}$ whose points may be put into correspondence in such a way that every geodesic on the one surface is pictured by a geodesic on the other. Apart from the trivial case where $S_{1}$ is isometric with (applicable on) $S$, or with a surface homothetic to $S$, the only pairs of surfaces obtained belong to the LiouviLLE class

or to the LiE class

$$
d s^{2}=(U+V)\left(d u^{2}+d v^{2}\right),
$$

$$
d s^{2}=(u+V) d u d v,
$$

the latter arising only with imaginary representations.

In this paper the problem is extended by requiring some, instead of all, the geodesics to correspond. The new problem is made precise by the following considerations :

In the first place, if only two simply infinite systems $\dagger$ of geodesics, on each surface, are to correspond, no restriction is thereby imposed on the pair of surfaces. In fact if $S$ and $S_{1}$ are taken arbitrarily, we may select any two systems of geodesics on each surface; if now we take these as parameter lines $u=$ const., $v=$ const., and let corresponding points be those represented by the same values of $(u, v)$, our requirement is satisfied.

On the other hand we have the

THEOREM. If in a point to point correspondence between two surfaces four simply infinite systems of geodesics on each surfuce correspond, then all geodesics necessarily correspond.

For the proof we suppose here, as in the rest of the paper, that the gaussian parameters on the two surfaces are selected so that corresponding points are represented by the same values of $(u, v)$, and let the length elements be

* Presented to the Society, October 31,1903 ; the results in 84 were presented separately August 31, 1903. Received for publication November 23, 1905.

† By a simply infinite system we mean one such that through each point of the region on the surface considered there passes one curve of the system and only one. 


$$
\begin{aligned}
& d s^{2}=E d u^{2}+2 F d u d v+G d v^{2}, \\
& d s_{1}^{2}=E_{1} d u^{2}+2 F_{1} d u d v+G_{1} d v^{2} .
\end{aligned}
$$

The geodesics are defined by differential equations of the form

$$
\begin{aligned}
& v^{\prime \prime}=A v^{\prime 3}+B v^{\prime 2}+C v^{\prime}+D, \\
& v^{\prime \prime}=A_{1} v^{\prime 3}+B_{1} v^{\prime 2}+C_{1} v^{\prime}+D_{1} .
\end{aligned}
$$

For corresponding geodesics, if any exist, these equations hold simultaneously, and therefore

$$
\left(A-A_{1}\right) v^{\prime 3}+\left(B-B_{1}\right) v^{\prime 2}+\left(C-C_{1}\right) v^{\prime}+\left(D-D_{1}\right)=0 .
$$

According to our hypothesis, this is satisfied by four different values of $v^{\prime}$; hence it must hold identically. This means that equations (2) coincide; hence all geodesics correspond and our theorem is proved.

The following corollaries deserve explicit statement:

If a surface can be mapped upon the plane in such a way that four systems of geodesics are pictured by straight lines, then all geodesics are so pictured, and hence the surface has constant curvature.*

If a point transformation of the plane converts four systems of straight lines into straight lines, then all straight lines are so converted, and the transformation is thus a collineation. $\dagger$

Our problem of "partial" geodesic representation can now be stated definitely : Determine all cases in which two surfaces correspond point by point so that there exist precisely three simply infinite systems of corresponding geodesics.

That this problem is of much greater generality than the DiNI problem is seen as follows. If three systems are to correspond, then equation (3) above must represent geodesics on each surface. If we express the fact that (3) satisfies say the first of equations (2) (the second is then necessarily satisfied also), we are led to a set of three partial differential equations of the second order in $E, F, G, E_{1}, F_{1}, G_{1}$. To satisfy these, $E, F, G$ may be taken at

* A special form of this proposition was obtained by Finsterwalder in his interesting report on the mechanics of surface deformation pablished in the $\mathrm{Jahresberichte}$ der deutschen Mathematiker-Vereinigung, vol. 6 (1899), p. 50 : If a surface contains four "linear systems" of geodesics, it must have constant curvature. This means here that there exist four equations of the form $a_{i} u+b_{i} v=$ const. $(i=1,2,3,4)$ which represent geodesics. This is equivalent to the requirement that. four systems of geodesics shall be pictured in the plane by four systems of parallel straight lines. The result is true, however, for any systems of straight lines. The special form is given in LilienthaL, Enoyklopädie der Mathematisoben Wissenschaften, vol. 3 (1903). p. 351 ; and STÄckeL, Mathematische Anualen, vol. 56 (1902), p. 502. In this paper Stäckel proposes the problem of determining all surfaces contain. ing three "linear systems" of geodesics and finds certain solutions.

†This result was given by the author in a note on The characterization of collineations, Bulletin American Mathematical Society, vol. 9 (1903), p. 545. 
random, and $E_{1}, F_{1}, G_{1}$ will still contain an infinite number of arbitrary constants. These constants may be selected so that (3) does not reduce to an identity, i. e., so that the surface $S_{1}$ is not merely isometric with a surface homothetic to $S$. Hence for any surface $S$ there exist an infinity of distinct surfaces $S_{1}$ satisfying the conditions of our problem.*

\section{§2. The confor mal problem.}

If the representation of $S_{1}$ on $S$ is conformal, the minimal lines correspond. This accounts for two simple systems of geodesics. The theorem of $\S 1$ then takes the form:

If a surface $S_{1}$ can be represented conformally on a surface $S$ in such a way that two real systems of geodesics, on each surface, correspond, then all geodesics correspond. By known theorems the surfaces must then, except for homothetic transformation, be isometric.

Our problem now is to determine all pairs of surfaces which admit of conformal representation with one real system of corresponding geodesics.

Since the representation is conformal, the length elements of both surfaces may be assumed in the isothermal form

$$
d s^{2}=e^{2 \omega}\left(d u^{2}+d v^{2}\right), \quad d s_{1}^{2}=e^{2 \omega_{1}}\left(d u^{2}+d v^{2}\right) .
$$

The geodesics are defined by

$$
v^{\prime \prime}=\left(\omega_{v}-v^{\prime} \omega_{u}\right)\left(1+v^{\prime 2}\right), \quad v^{\prime}=\left(\omega_{1 v}-v^{\prime} \omega_{1 u}\right)\left(1+v^{\prime 2}\right) .
$$

Geodesics on either surface which correspond to geodesics on the other must satisfy both these equations; hence, disregarding the factor $1+{v^{\prime}}^{2}$ leading to minimal lines, we find

where

$$
\delta_{v}-v^{\prime} \delta_{u}=0
$$

The condition that (6) shall define geodesics is found to be

$$
\delta_{u} \delta_{v}\left(\delta_{u u}-\delta_{v v}\right)-\delta_{u v}\left(\delta_{u}^{2}-\delta_{v}^{2}\right)=\left(\omega_{u} \omega_{1 v}-\omega_{v} \omega_{i u}\right)\left(\delta_{u}^{2}+\delta_{v}^{2}\right),
$$

which, as should be the case, is unaffected by the interchange of $\omega$ and $\omega_{1}$. It is easy to verify that (8) is also the condition that the curves $\delta=$ const. on either $S$ or $S_{1}$ shall be (geodesically) parallel. When this is the case the system of geodesics $(6)$ consists of the orthogonal trajectories of these parallels.

\footnotetext{
${ }^{*}$ A given element $d s^{2}=E d u^{2}+2 F d u d v+G d v^{2}$, of course, defines not one surface but a class of isometric surfaces ; it is, however, unnecessary to distinguish between the members of such a class since our problem deals with the internal geometry of surfaces.
} 
Hence all solutions $S, S_{1}$, of the conformal problem may be obtained as follows: Let $S$ be any surface assumed given in the isothermal form

$$
d s^{2}=e^{2 \omega}\left(d u^{2}+d v^{2}\right)
$$

then the related surfaces $S_{1}$ are of the form

$$
d s_{1}^{2}=e^{2(\omega+\delta)}\left(d u^{2}+d v^{2}\right),
$$

where $\delta$ is any function of $u, v$, such that the curves $\delta=$ const. on $S$ are parallel. By the theory of parallels, $\delta$ is therefore an arbitrary function of $\phi$, where $\phi$ is the general solution of

$$
\phi_{u}^{2}+\phi_{v}^{2}=e^{2 \omega} .
$$

If the finite equation of the geodesics on $S$ is known, then all related surfaces $S_{1}$ may be found by quadratures. This follows from the known theorem that the determination of the orthogonal trajectories of a given system of geodesics depends upon quadratures.

In particular, the result applies to the determination of surfaces which can be mapped conformally on the plane so that a system of geodesics is pictured by straight lines. All such surfaces are given by

$$
d s^{2}=e^{2 \omega}\left(d u^{2}+d v^{2}\right)
$$

where the function $\omega$ is such that the curves $\omega=$ const. in the $u, v$ plane are parallel. The explicit result is that $\omega$ is an arbitrary function $f$ of the argument

$$
\phi=\int \frac{d v-t d u}{\sqrt{1+t^{2}}}
$$

where $t$ is defined by any equation of the form

$$
u+v t=\psi(t) .
$$

Thus $\omega$ involves two arbitrary functions $f$ and $\psi$.

\section{§3. Geodesic paraneters.}

Another analytical representation of the surface $S, S_{1}$, which is more convenient for the problem discussed in $\S 4$, may be obtained by using gaussian geodesic parameters. On each of the surfaces let the assumed system of geodesics be $v=$ const., and let their orthogonal trajectories, which also correspond since the representation is conformal, be $u=$ const. Then the length elements take the form

$$
\begin{aligned}
& d s^{2}=E(u) d u^{2}+G(u, v) d v^{2}, \\
& d s_{1}^{2}=E_{1}(u) d u^{2}+G_{1}(u, v) d v^{2} .
\end{aligned}
$$


The condition for conformality is

$$
\frac{G_{1}}{G^{\frac{1}{y}}}=\frac{E_{1}}{E}
$$

By a proper selection of the parameter $u$ the function $E$ may be reduced to unity. We may then write

$$
\begin{aligned}
& d s^{2}=d u^{2}+G d v^{2}, \\
& d s_{1}^{2}=U\left(d u^{2}+G d v^{2}\right),
\end{aligned}
$$

where $G$ is any function of $u, v$ and $U$ any function of $u$.

All pairs of surfaces $S, S_{1}$ which admit of conformal representation with a real system of corresponding geodesics may be reduced to the form (10). The converse also holds.

If any surface $S$ is given with an assigned system of geodesics on it, there exist an infinite number of related surfaces $S_{1}$ depending upon an arbitrary function $U$.

In any conformal representation the ratio of corresponding length elements, $\rho=d s_{1} / d s$, is independent of the direction of those elements and is thus a point function. In our case we have, from (10),

$$
\begin{aligned}
& d s_{1} \\
& d s
\end{aligned}=\sqrt{U}
$$

Since the curves $u=$ const. are parallel, we have the result:

In a conformal representation of the kind considered, the curves, on either surface, for which the ratio $d s_{1} / d s$ is constant are geodesicully parallel.

The converse, which may be proved without much difficulty, takes the form:

If in any conformal representation the curves $d s_{1} / d s=$ const. are parallel on one surface, they are also parallel on the other surface; the geodesics orthogonal to these parallels then correspond by the representation.

We now determine the surfaces $S_{1}$ which can be represented on a plane. The element $d s^{2}$ in (10) nust have zero curvature. From this we find that $G$ must have the form

$$
G=\left(V_{1} u+V\right)^{2},
$$

where $V, V_{1}$ are any functions of $v$.

Therefore all cases of surfaces $S_{1}$ which can be conformally represented on a plane $S$ so that $\infty^{1}$ geodesics are pictured by straight lines are given by

$$
\begin{aligned}
& d s^{2}=d u^{2}+\left(V_{1} u+V\right)^{2} d v^{2}, \\
& d s_{1}^{2}=U\left\{d u^{2}+\left(V_{1} u+V\right)^{2} d v^{2}\right\} .
\end{aligned}
$$

If $V_{1}=0$ and $V=1$, the element $d s_{1}$ belongs to a surface of revolution. Thus the surfaces applicable on surfaces of revolution enter as particular solutions of our problem. 
§4. A class of conformal transformations in the plane.

The case in which both $S$ and $S_{1}$ are planes is especially interesting. The question is then to determine all conformal representations of a plane on itself (or on another plane) by which a simply infinite system of straight lines are pictured by straight lines. By the theorem of $\S 1$, there cannot be two real systems unless the representation reduces merely to a conformal collineation, i. e., a similitude transformation.

All cases where $S$ has zero curvature are included in (11); the question is then to determine the functions $U, V, V_{1}$, so that $S_{1}$ shall have zero curvature also.

We divide the discussion according to the vanishing or non-vanishing of $V_{1}$.

If $V_{1}=0$, the elements become, after a proper change in the parameter $v$ and the substitution of $U^{2}$ for $U$,

$$
d s^{2}=d u^{2}+d v^{2}, \quad d s_{1}^{2}=U^{2}\left(d u^{2}+d v^{2}\right) .
$$

The condition that the curvature of $d s_{1}$ shall vanish is

hence

$$
U U^{\prime \prime}-U^{\prime 2}=0 \text {; }
$$

The solution obtained is

$$
U=e^{a u+b} .
$$

$$
d s^{2}=d u^{2}+d v^{2}, \quad d s_{1}^{2}=e^{2(a u+b)}\left(d u^{2}+d v^{2}\right) .
$$

To find the corresponding conformal transformations, we reduce (13) to the minimal form by putting

This gives

$$
d \alpha=d u+i d v, \quad d \beta=d u-i d v .
$$

$$
d s^{2}=d \alpha d \beta, \quad d s_{1}^{2}=e^{a(\alpha+\beta)+b} d \alpha d \beta .
$$

Now every conformal transformation is equivalent to a substitution of some function $f(\alpha)$ for $\alpha$ and some function $g(\beta)$ for $\beta$. The condition that $d s^{2}$ is converted into $d s_{1}^{2}$ is

$$
f^{\prime}(\alpha) g^{\prime}(\beta)=e^{a(\alpha+\beta)+b} .
$$

The solution of this functional equation is

$$
f=e^{c_{1} a+a_{1}}+a_{2}, \quad g=e^{c_{1} \beta+b_{1}}+b_{2} .
$$

The second case, where $V_{1}$ is not zero, is more complicated. The elements (11) may now be reduced to

$$
d s^{2}=d u^{2}+(u+V)^{2} d v^{2}, \quad d s_{1}^{2}=U^{2}\left\{d u^{2}+(u+V)^{2} d v^{2}\right\} .
$$

The condition that $S_{1}^{y}$ has zero curvature is

$$
U U^{\prime}+(u+V)\left(U U^{\prime \prime}-U^{\prime 2}\right)=0 .
$$


The case where $U U^{\prime \prime}-U^{\prime 2}$ vanishes is trivial since (16) shows that then $U$ must be a constant; then we have merely a similitude transformation.

We may therefore write (16) in the form

$$
\frac{U U^{\prime}}{U U^{\prime \prime}-U^{\prime 2}}+u=-V
$$

Each member of this equation must reduce to the same constant; this may without loss of generality be assumed zero. We find

so that the new solution is

$$
V=0, \quad U=a u^{c}
$$

$$
d s=d u^{2}+u^{2} d v^{2}, \quad d s_{1}^{2}=a^{2} u^{2 c}\left(d u^{2}+u^{2} d v^{2}\right) .
$$

The reduction to minimal parameters, by means of the substitution

gives

$$
d \alpha=e^{i v}(d u+i u d v), \quad d \beta=e^{-i n}(d u-i u d v),
$$

$$
d s^{2}=d \alpha d \beta, \quad d s_{1}^{2}=a \alpha^{c} \beta^{c} d \alpha d \beta .
$$

The equation for the determination of $f$ and $g$ is now

$$
f^{\prime}(\alpha) g^{\prime}(\beta)=a \alpha^{c} \beta^{c} \text {. }
$$

The solutions are found to be of these two types:

$$
\begin{array}{cl}
f=a_{1} \alpha^{c_{1}}+a_{2}, & g=b_{1} \beta^{c_{1}}+b_{2} ; \\
f=a_{1} \log \alpha+a_{2}, & g=b_{1} \log \beta+b_{2} .
\end{array}
$$

We thus find that all conformal transformations of the kind required are given by (15), (20) and (21). For real transformations $f$ and $g$ must be coniugate, so that it is sufficient to consider say $f$. Using the usual complex variable $z$ instead of $\alpha$, we find that the real transformations are

$$
Z=e^{a x+b}, \quad Z=a \log z+b, \quad Z=a z^{k}+b,
$$

where $a, b$ are any (real or complex) constants and $k$ is real. By means of similitude transformation these may be reduced to the canonical forms

$$
Z=e^{z}, \quad Z=\log z, \quad Z=z^{k} .
$$

Every real conformal transformation of the plane into itself which converts a single infinity of real straight lines into straight lines belongs to one of the three types (23). In the exponential type a set of parallels is converted into a pencil (with a finite vertex); in the logarithmic type a pencil is converted into a set of parallels; in the monomial type a pencil is converted into a pencil.

The first two types may be regarded as limiting cases of the last type. Columbia University. 